\title{
Modelo de transferencia-apropiación de un
}

sistema de producción integral de alimentos

(Sipia) para el desarrollo humano*

\section{Technology Transfer-ownership Model of an Integrated System of Food Production (Sipia) for Human Development}

\section{Modèle de transfert-appropriation d'un système de production intégral de nourriture (Sipia) pour le développement humain}

\author{
Ma.-Luisa Isla-Esquivel ${ }^{* *}$, Alfonso Cuevas-Jiménez ${ }^{* * *}$, Javier Aranda****
}

Recibido: 2015-06-12 // Aprobado: 2015-07-12 // Disponible en línea: 2016-01-30

Cómo citar este artículo: Isla-Esquivel, M. L., Cuevas-Jiménez, A. y Aranda, J. (2016). Modelo de transferencia-apropiación de un sistema de producción integral de alimentos (Sipia) para el desarrollo humano. Ambiente y Desarrollo, 20 (38), 99-110. http://dx.doi.org/10.11144/Javeriana.ayd20-38.mtas doi: 10.11144/Javeriana.ayd20-38.mtas

\begin{abstract}
Resumen
Se presenta un modelo de transferencia-apropiación tecnológica que tiene como objetivo la conformación de comunidades de trabajo-aprendizaje en torno a un sistema de producción integral de alimentos, con el fin de formar habilidades para la vida que impulsen el desarrollo humano. Lo integran tres ejes que se trabajan de manera interrelacionada: el eje técnico, el pedagógico y el de desarrollo humano. Dicha propuesta surge de la necesidad de avanzar de un enfoque basado solo en el proceso de transferencia a uno que incluya la apropiación, en el cual convergen tres conceptos importantes para el ser humano: el trabajo como proceso de promoción del desarrollo, la educación como vía para la formación de la persona y el concepto de naturaleza, vinculado orgánicamente a los seres humanos. El modelo que se presenta, desarrollado en la Universidad Marista de Mérida (México), busca integrar las cuatro funciones sustantivas de la institución: docencia, investigación, extensión y servicio y desarrollo comunitario.
\end{abstract}

Palabras clave: transferencia-apropiación; comunidad de trabajo-aprendizaje; producción integral; desarrollo humano; habilidades para la vida

\footnotetext{
Este artículo es producto de la línea de investigación Educación experiencial y procesos de transferencia de tecnologías, desarrollada por María Luisa Isla Esquivel en el periodo 2013-2015.

** Maestra en Educación, Universidad Marista de Mérida, México. Correo electrónico: misla@marista.edu.mx.

*** Doctor en Ingeniería, Universidad Marista de Mérida, México. Correo electrónico: acuevas@marista.edu.mx.

**** Maestro en Administración de Negocios Acuícolas, Universidad Marista de Mérida, México. Correo electrónico: jaranda@marista.edu.mx.
} 


\begin{abstract}
A Technology Transfer-Ownership model is presented, which aims to forming worklearning communities associated with an integrated system of food production in order to create life skills which promote human development. It consists of three axes which work in an interrelated manner: the technical axis, the pedagogical axis and the human development axis. This proposal arises from the need to move from an approach which is solely based on the transfer process towards one which includes ownership, where three important concepts converge: work in the form of promoting development; education as a means for training people and the concept of nature, organically linked to human beings. The model presented here was developed at the Marist University of Merida (México), which allows four fundamental functions of the institution to be integrated: teaching, research, extension and service and community development.
\end{abstract}

Keywords: transfer-ownership; work-learning community; integrated production; human development; life skills

\title{
Résumé
}

On présente un modèle de transfert-appropriation technologique qui a en tant qu'objectif la conformation de communautés de travail-apprentissage par rapport à un système de production intégrale de nourriture, avec l'objectif de façonner les habilités pour la vie qui renforcent le développement humain.

Le modèle est constitué par trois axes qui travaillent d'une façon interconnecté : l'axe technique, pédagogique et celui du développement humain. Cette proposition naît du besoin de passer d'une perspective fondée seulement dans le processus de transfert à une qui inclut l'appropriation, dans laquelle confluent les trois concepts importants pour l'être humain : le travail en tant que processus de renforcement du développement, l'éducation en tant qu'une voie pour la formation de la personne et le concept de nature, rapporté organiquement aux êtres humains. Le modèle qui se présente, développé à l'Université Marista de Mérida (Mexique), cherche intégrer les quatre fonctions substantives de l'institution : enseignement, recherche, extension et service et développement communautaire.

Mots-clés: transfert-appropriation ; communauté de travail-apprentissage ; production intégrale ; développement humain ; habilités pour la vie 


\section{Introducción}

El compromiso por el servicio, especialmente a los más necesitados, es uno de los rasgos que distinguen a la Universidad Marista de Mérida (UMM). Está plasmado en su misión institucional, al proponerse la formación académica, humana y cristiana de los jóvenes universitarios, con el carisma educativo marista, en una tarea compartida por hermanos y laicos, a través no solo de las tres funciones sustantivas que toda institución de educación superior atiende — docencia, investigación y extensión—, sino que cuenta con una más, explícitamente orientada al "servicio y desarrollo comunitario".

En la fundación de la umM en 1996, el desafío quedó claramente planteado: "atreverse a generar proyectos realistas de nación, en los que estén presentes alternativas a corto, mediano y largo plazo que permitan una estructura más humana y por tanto más moderna" (Cavazos, 1997, p. 7). El indicador del logro fue contundente:

El termómetro será cuando nuestros pobres, nuestro pueblo maya, pobladores legítimos de estas tierras, den muestras de mejoría social y económica, cuando puedan con hechos constatar que han salido de sus penurias, porque los estudiantes, catedráticos, ciudadanía y gobierno se han ocupado de la justicia social. Entonces será bien útil nuestra Universidad. (Cavazos, 1997, p. 7)

El desafío exigía un trabajo coordinado entre las funciones sustantivas ya mencionadas, encaminado a promover la construcción y apropiación de un conocimiento socialmente responsable, así como la orientación de las profesiones hacia la promoción del desarrollo humano sostenible. En el ámbito académico internacional, la Organización de las Naciones Unidas para la Educación, la Ciencia y la Cultura (Unesco) ha convocado a las instituciones de educación superior a canalizar la influencia que ejercen en la sociedad, orientándola hacia la transformación de los ámbitos de vulnerabilidad social y económica presentes en nuestras sociedades (De la Cruz y Sasia, 2008).

En este contexto, se plantea que la investigación científica que realice la UмM sea de naturaleza aplicada, orientada a resolver problemas e identificar oportunidades de desarrollo. En el año 2001, la Escuela de Administración de Recursos Naturales de la UMM puso en marcha la Unidad Experimental Marista (Unexmar), área destinada a la experimentación y el manejo integral de recursos naturales, donde se asesora y capacita a estudiantes, académicos y a la comunidad en general, a través de modelos demostrativos-prácticos que fomenten el desarrollo sustentable. Uno de los objetivos de esta unidad fue diseñar y evaluar estrategias de optimización tecnológica en la producción diversificada, a partir de los recursos naturales disponibles localmente. Bajo principios agroecológicos, se desarrolló un sistema de producción integral de alimentos (Sipia), disponible como un paquete tecnológico para mejorar la productividad del suelo, manteniendo un equilibrio ambiental con cosechas que satisfagan la necesidad de una alimentación sana y favorezcan la economía de los participantes. Este sistema reutiliza los residuos orgánicos derivados de los componentes agrícola, acuícola y pecuario que lo constituyen, colocándolos en un flujo de energía y reciclaje de nutrientes, reduciendo así la dependencia de insumos externos (Benbrook, 1991; Porcuna, 2007; Flores, 2001; Roncal Cárdenas, 2010).

La experiencia adquirida en diez años de producción sostenida en el Sipia, da solidez al paquete tecnológico propuesto. Se diseña entonces una estrategia para que el Sipia, además de resolver problemas, identifique oportunidades de desarrollo que generen proyectos realistas, capaces de promover mejoría social y económica en grupos vulnerados. Se desarrolla un modelo de transferencia/apropiación que atienda los procesos de capacitación y de organización como elementos fundamentales (Isla-Esquivel et al., 2011).

Este documento tiene como objetivo presentar los enfoques teóricos en los que se sustenta este modelo de transferencia/apropiación, así como las fases para su implementación. 


\section{Modelo de transferencia-apropiación}

El modelo de transferencia-apropiación que aquí se presenta, tiene como objetivo la conformación de una comunidad de trabajo-aprendizaje en torno al manejo de un sistema de producción integral de alimentos, como herramienta para el desarrollo humano.

Esta propuesta se sustenta en las siguientes premisas:

a) El grupo al cual se transfiere-apropia el paquete tecnológico está integrado por sujetos responsables de su propio desarrollo.

b) Participar en proyectos sociales orientados a promover el desarrollo humano facilita el empoderamiento de los participantes, el aprovechamiento de sus fortalezas y la minimización de los riesgos que los rodean, mediante el fomento de factores protectores como las habilidades para la vida.

c) El paquete tecnológico del Sipia contribuye a una alimentación sana, favorece la economía de los participantes y promueve el desarrollo sustentable.

d) El modelo de transferencia-apropiación es una estrategia formativa basada en un tipo de vinculación universidad-comunidad para la participación con responsabilidad social y servicio a los demás.

El modelo está integrado por tres ejes: eje técnico, eje pedagógico y eje de desarrollo humano. El eje pedagógico alimenta al modelo con una visión transformadora de la educación, el eje de desarrollo humano orienta el proyecto hacia la construcción de comunidades solidarias que trabajan por el bien común y el eje técnico potencia, a través del trabajo-aprendizaje, la integración de los dos ejes anteriores. La condición para el logro del proceso de transferencia-apropiación radica en la necesaria interrelación entre los tres ejes, base fundamental del modelo.

\section{Eje técnico}

Los sistemas integrales de producción tienen como principio la relación intrínseca y holística entre sus componentes o subsistemas, cuyos subproductos o desechos generados son reutilizados y aprovechados de manera recíproca, permitiendo un comportamiento eficiente de materia y energía. La transferencia vertical de nutrientes de un subsistema a otro minimiza su pérdida, incrementa la eficiencia y en consecuencia tiende a la autosustentabilidad (Flores, 2001). Este tipo de sistema de producción utiliza un bajo nivel de insumos externos y a su vez genera sinergia dentro de la producción, mejorando la eficiencia productiva y contribuyendo así al desarrollo sostenible (Pullin, 2003).

Algunos de los ejemplos que se han reportado en la literatura se enfocan a la integración de la agricultura y la acuicultura, como es el caso de Asia e India empleando carpas (Cyprinus carpio) y bagres (Clarias batrachus) en plantaciones de arroz desde hace cientos de años (Phong et al., 2007). En China y Tailandia se implementaron formas de integración más complejas, incluyendo patos, peces y hortalizas, adecuándose posteriormente en América Latina con resultados alentadores (Flores, 2001). Murshed-E-Jahan y Pemsl (2011) describen el impacto del establecimiento de sistemas acuiculturaagricultura integrada, donde la productividad total resulta en un mayor consumo de alimentos y una mejor nutrición para los hogares de los productores. Brummett y Williams (2000) sostienen que un número cada vez mayor de pequeños agricultores han venido adoptando y adaptando la acuicultura a sus sistemas agrícolas existentes y aumentando poco a poco su eficiencia productiva. Este enfoque, basado en la participación local y la transferencia de tecnología, demostraría que estos sistemas de producción pueden ser más productivos y sostenibles — ambiental y socialmente - en el largo plazo.

El eje técnico del modelo que aquí se presenta, hace referencia al paquete tecnológico Sipia, el cual contempla el desarrollo de acciones enfocadas al cultivo agropecuario, desde el manejo de un sistema de producción integral de alimentos bajo principios agroecológicos. Su alcance no se limita a la acción 
per se de cultivar, sino que se extiende a aprovechar sustentablemente los recursos naturales desde una perspectiva tradicional y regional, adecuada al contexto sociocultural, ecológico y económico del participante. Este paquete consta de cuatro componentes principales (figura 1): 1) ave-acuícola, 2) agri-acuícola, 3) pecuario, 4) abonos orgánicos, en un área de $2500 \mathrm{~m}^{2}$ con una infraestructura que comprende un corral de patos sobre un estanque acuícola extensivo, área agrícola, corral de aves de traspatio y un área de compostaje. Los distintos componentes del sistema se encuentran relacionados entre sí, a través del aprovechamiento y el manejo de los insumos y desechos de cada componente. El Sipia tiene la capacidad de producir más de cuarenta variedades distintas de hortalizas, granos y frutas, carne y huevo de dos variedades de aves de corral y de pato criollo, carne de pescado de agua dulce, manteniendo la constante productividad del suelo a través de abonos orgánicos. En conjunto, el sistema puede garantizar, aproximadamente, un volumen de producción de 28 kilogramos de alimento a la semana, equivalentes a 1,5 toneladas anuales.

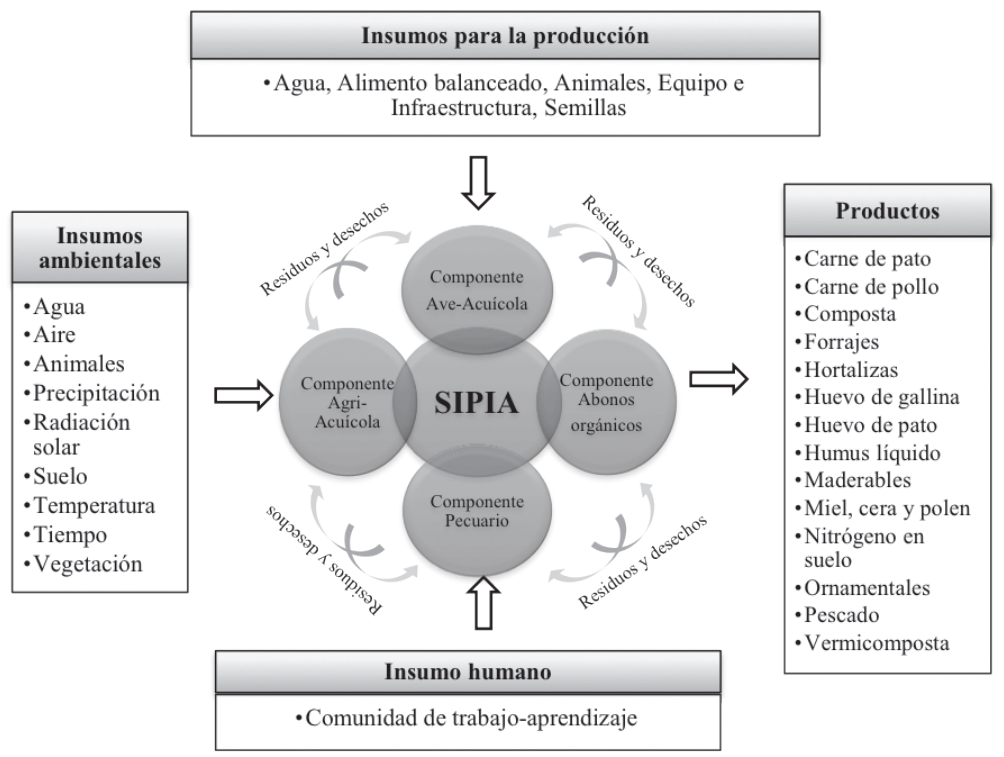

Figura 1. Desempeño del sistema de producción integral de alimentos

Fuente: elaboración propia

\section{Eje pedagógico}

El eje pedagógico de este modelo es considerado la bisagra entre el eje técnico y el eje de desarrollo humano, ya que es a través de la mirada humanizadora de la educación que se propone entrelazar las lecciones de la naturaleza con las del grupo, orientándolas hacia la plena realización de la persona mediante su participación en el trabajo colectivo.

La educación en este eje se entiende como una educación para la vida, comprometida con el pleno desarrollo de la persona y su formación en un sentido amplio, de tal manera que abone a su preparación para la vida en sociedad. Mejía y Awad (2003) afirman que el acto educativo es ante todo un hecho ético que busca el empoderamiento de los excluidos y desiguales.

Un supuesto básico en el que descansa este eje es que "el conocimiento es situado, es parte y producto de la actividad, del contexto y de la cultura en que se desarrolla y utiliza" (Díaz-Barriga, 2006, p. 19), por lo que hay que evitar caer en las tradiciones de la educación bancaria, pues pondrían en riesgo el espíritu del modelo al desviarlo hacia un entrenamiento mecánico en el uso de una técnica determinada. 
Un pilar teórico en el que se apoya este modelo es la educación popular, desde la cual se utiliza el concepto compuesto de "transferencia-apropiación", para diferenciarlo de los términos "transferencia", "capacitación" o "extensión", entendidos como el acto de extender contenidos técnicos a quienes no los tienen. La transferencia-apropiación se entiende en el sentido que Freire le da a la capacitación, como el acto por el cual el proceder técnico se ofrece al educando como un problema, al cual debe responder, y no solo aceptar como si se tratara de algo terminado. El autor afirma que "En el proceso de aprendizaje, sólo aprende verdaderamente aquel que se apropia de lo aprendido, transformándolo en aprehendido, con lo que puede, por eso mismo, reinventarlo, llegando a ser capaz de aplicar lo aprendido-aprehendido, a las situaciones existenciales concretas" (Freire, 1973, p. 28). Es por ello que el primer concepto que se enuncia en el objetivo del Modelo de Transferencia-Apropiación de Tecnología para el desarrollo humano, es el de la conformación de una comunidad, pues la educación es comunicación, es diálogo, en la medida en que no es la transferencia mecánica del saber, sino un encuentro de sujetos interlocutores: "El diálogo, es el encuentro amoroso de los hombres que, mediatizados por el mundo lo transforman y, transformándolo, lo humanizan" (Freire, 1973, p. 46). Solo en una comunidad que reconoce la capacidad de sus miembros para comunicarse entre sí a nivel intelectivo, se posibilita la creación de conocimiento y la organización del trabajo que los potencie como seres humanos (Gelvis y Useche, 2009).

Otro pilar teórico del modelo es la metodología de trabajo-aprendizaje (MT-A) propuesta por Duch (2009), que debe entenderse como una alternativa pedagógica al enfoque de la capacitación, caracterizado por separar esta, artificialmente, de los problemas concretos de trabajo, reduciéndola en consecuencia al adiestramiento manual de los productores en una técnica específica. En su lugar, la MT-A tiene como eje central de la actividad capacitadora la reflexión y el análisis del trabajador acerca de sus tareas, para lo cual se requiere que los procesos de capacitación estén orgánicamente relacionados con las necesidades de los productores responsables del funcionamiento de la organización laboral. En la MT-A el trabajo es considerado un proceso de promoción del desarrollo con una perspectiva de autogestión y realización individual y social. Esta metodología de formación en y para el trabajo procura la plena realización de la persona, al fijar como su propósito más alto el desarrollo de la conciencia crítica, la comprensión sobre los fines de la vida, el sentido de solidaridad y el compromiso en la construcción de un mundo más humano (Duch, 2009).

En el modelo de transferencia-apropiación, el objeto de estudio de la comunidad de trabajoaprendizaje es el proceso de trabajo que reúne a los participantes, y al ser el conocimiento parte y producto de la actividad, el diálogo, la reflexión y el análisis son los procesos básicos para la capacitación; por tanto, es necesario recurrir a otro pilar teórico que oriente estos procesos reflexivos. El concepto de "práctica reflexiva" de Schön (1992) propone que expertos y novatos en una actividad determinada formen una comunidad de indagación, en la cual, de manera colectiva y dialógica, reflexionen sobre sus maneras de actuar y descubran el conocimiento tácito o "conocimiento en la acción" desde el cual proceden, así como las innovaciones a las que recurren cuando ese conocimiento no los conduce a las metas esperadas. Esta reflexión en la acción es como si se estableciera una conversación reflexiva entre la persona y lo que ocurre en una determinada situación, permitiéndole reorganizar lo que está haciendo mientras lo está haciendo. También se genera conocimiento nuevo cuando el tipo de reflexión que hace el grupo es posterior a las acciones tomadas sobre la marcha, a la cual Schön le llama reflexión sobre la reflexión en la acción.

El reconocimiento de la existencia de situaciones singulares, inciertas y conflictivas en el ejercicio de las profesiones y oficios requiere construir una cultura de la práctica reflexiva en una comunidad de indagación, pues propicia aprender del otro, quien puede aportar ideas e iniciativas que sirvan para encontrar soluciones innovadoras a los problemas, así como generar una teoría o un conocimiento que permita responder a las cambiantes situaciones prácticas (Cassís, 2011).

En resumen, el modelo de transferencia-apropiación que aquí se presenta es una propuesta de enseñanza situada no formal, centrada en las necesidades de los sujetos y en el aprendizaje, entendido este último como un proceso de construcción de significados a través del diálogo en grupo. 


\section{Eje de desarrollo humano}

En el objetivo del modelo de transferencia-apropiación de tecnología que aquí se presenta, está claramente planteado que su meta última es la promoción del desarrollo humano en los participantes. Un desarrollo centrado en la persona y no en los objetos. Un desarrollo en el que los participantes tengan un papel protagónico en el proceso, que se refiera a aspectos fundamentalmente humanos, en contraste con el que tiene como meta alcanzar los niveles materiales de vida de quienes viven en países más industrializados, que tenga como finalidad elevar la calidad de vida de los participantes.

Ya desde finales del siglo pasado se afirmaba que el paradigma del desarrollo que había guiado los pasos hacia el crecimiento en América Latina estaba en crisis, no solo económica, sino también política, cultural y social, lo que condujo a la pérdida de la capacidad de soñar con construir alternativas a partir de medios propios (Max-Neef, 1993).

Otro crítico de la economía del bienestar tradicional que propuso un nuevo enfoque del desarrollo fue Sen, quien afirmó que "A pesar de que la opulencia mundial ha experimentado un aumento sin precedentes, el mundo contemporáneo niega libertades básicas a un inmenso número de personas, quizá incluso a la mayoría" (2000, p. 3).

A inicios del siglo xxi el cuestionamiento al desarrollo convencional se vio reforzado, reconociendo que no daba respuestas adecuadas al deterioro ambiental ni al agotamiento ecológico del planeta. La Tierra no tiene la capacidad de absorción y resiliencia para que todos repitan el consumismo propio de los países industrializados (Gudynas y Acosta, 2011).

En este contexto de "cansancio" con las ideas convencionales sobre el desarrollo, han surgido alternativas para la creación de "otro desarrollo", entre las cuales se puede citar el desarrollo a escala humana, el florecimiento humano, el buen vivir, o el desarrollo como libertad, por mencionar algunas. Entre las premisas sobre las cuales descansan estas alternativas se pueden mencionar las siguientes: el desarrollo es de las personas no de las cosas; al ser humano hay que verlo completo - con todas sus necesidades y capacidades-; la persona o el grupo de personas tienen un papel protagónico en la construcción de su propio desarrollo y del ajeno; el rol de lo material es instrumental, no es el fin del desarrollo (Boltvinik, 2005; Gudynas, 2011; Max-Neef, 1993; Sen, 2000).

Sin embargo, para poder participar en este tipo de desarrollo se requiere la construcción de ciertas habilidades. En 1990 el Programa de las Naciones Unidas para el Desarrollo (PNUD) definió el desarrollo humano centrándose en las personas, proponiendo como su objetivo la formación de capacidades humanas, tales como un mejor estado de salud o mayores conocimientos. Así, afirma que si el desarrollo humano no logra equilibrar la formación y utilización de las capacidades humanas, una buena parte del potencial de los individuos se verá frustrada. Las capacidades humanas son reconocidas como la riqueza de las personas, o su pobreza en caso de no tenerlas, por lo que muchas organizaciones dedicadas al desarrollo humano se han orientado a trabajar en su promoción. Aspecto clave de este enfoque es la adquisición de habilidades sociocognitivas y emocionales para enfrentar y superar las múltiples situaciones de adversidad y avanzar en la mejora de su calidad de vida. Surge entonces el enfoque de Habilidades para la Vida $(\mathrm{HpV})$ que las define como "las habilidades para el comportamiento positivo y adaptable que permiten a los individuos lidiar eficazmente con las demandas y los retos de la vida cotidiana” (Organización Mundial de la Salud (oms), 1993). 
Esta organización considera fundamentales en todas las culturas diez habilidades para la vida:
1. Autoconocimiento
2. Empatía
3. Comunicación asertiva
4. Relaciones interpersonales
5. Toma de decisiones

6. Solución de problemas y conflictos

7. Pensamiento creativo

8. Pensamiento crítico

9. Manejo de emociones y sentimientos

10. Manejo de tensiones y estrés

Las habilidades para la vida se consideran destrezas que facilitan apropiar y poner en acción el conocimiento, las cualidades y los valores. El conocimiento del entorno y los contextos en los que se desempeña la persona son el marco para su desarrollo, dado que estas destrezas tienen aplicación en el ámbito de las acciones y relaciones del sujeto consigo mismo y con los otros a través de la interacción social. A la hora de elaborar programas de intervención se sugiere tomar en cuenta tres características importantes de estas habilidades (Mantilla Castellano y Chahín Pinzón, citados por Crabay, 2010):

a) La especificidad para cada cultura,

b) Que sean genéricas, y

c) Que no sean únicas o exclusivas para cada situación o problema.

El enfoque actual de las $\mathrm{HpV}$, además de estar orientado tanto al bienestar humano como al social, también lo está a la transformación del entorno; a optar por la vida individual y colectiva; por la vida fisiológica y espiritual, así como por la vida humana y en armonía con la naturaleza (Martínez, 2014).

En este modelo de transferencia-apropiación de tecnología, las $\mathrm{HpV}$ son abordadas de una manera integrada al proceso de transferencia, sobre la práctica, en comunidad de trabajo-aprendizaje, orientadas a promover el desarrollo humano de los integrantes del proyecto y de la comunidad que se construye en torno a este, con la intención de fomentar el desarrollo en el presente y construir condiciones para hacerlo en el futuro con las siguientes generaciones, de manera que promuevan un desarrollo humano sostenible. Las habilidades que se trabajan principalmente en este modelo son las siguientes:

Habilidades para trabajar en equipo de forma organizada. Buscan que los integrantes tengan claramente identificadas las diferentes tareas que requiere una buena atención del Sipia, que las distribuyan de manera equitativa y justa y las atiendan en tiempo y forma. También promueven el apoyo solidario entre los participantes, así como una adecuada y asertiva comunicación entre ellos.

Habilidades de reflexión y toma de conciencia. El desarrollo de esta habilidad consiste en que los integrantes del equipo logren hacer descripciones objetivas tanto de sucesos técnicos como de los procesos grupales, sobre los cuales identifiquen el verdadero problema, los recursos con los que se cuenta, diferentes alternativas de solución y sus respectivas consecuencias, a fin de crear colectivamente estrategias de acción adecuadas, pertinentes y eficaces.

Habilidades participativas. Los tipos de participación que se promueven entre los integrantes del grupo son la incluyente y la transformadora, de acuerdo con la clasificación propuesta en la Escalera de la Participación (Oficina de Derechos Humanos Arzobispado de Guatemala, 2014), desarrollando habilidades que les permitan expresar su punto de vista e involucrarse en la realización de acciones/ tareas/proyectos propuestos a partir del análisis de su realidad concreta y con fines del bien común. En caso de que los participantes sean niños, niñas y/o adolescentes, los niveles de participación que se promueven son 6, 7 y 8 de la Escalera de la Participación (Hart, citado por Alfageme et al., 2003).

Habilidades para la convivencia armónica y la solución pacífica de conflictos. Otro grupo de habilidades necesarias para la vida es el de aquellas que permiten una convivencia armónica y la solución pacífica de los conflictos. El diálogo auténtico entre las personas requiere una comunicación objetiva 
y precisa para aclarar situaciones, el uso de tonos pacíficos y conciliadores, la asertividad necesaria para hacer valer los derechos propios respetando los ajenos, así como el establecimiento de relaciones amables, empáticas y solidarias con los demás.

\section{Fases de apropiación del Sipia}

El logro de la apropiación del Sipia depende en gran medida de que los involucrados en el proyecto verdaderamente se vayan convirtiendo en una comunidad de trabajo-aprendizaje. Para la implementación de este modelo se proponen cinco fases que incluyen una serie de procesos que llevan a la consolidación de la apropiación del sistema de producción. En la tabla 1 se describen de manera general las fases, durante las cuales se trabajan de manera entrelazada los ejes que integran el modelo: técnico, pedagógico y de desarrollo humano, a través de la reflexión colectiva sobre el proceso de trabajo, como ha sido explicado anteriormente.

Tabla 1. Fases de la transferencia-apropiación del Sipia

\begin{tabular}{|c|c|c|}
\hline Fases & Descripción & Generalidades \\
\hline Primera & $\begin{array}{l}\text { Conocimiento mutuo entre } \\
\text { quien inicia la transferencia y } \\
\text { quien se apropia, para llegar } \\
\text { a compromisos y acuerdos }\end{array}$ & $\begin{array}{l}\text { El grupo que expresa su interés en el Sipia se acerca a la } \\
\text { institución que promueve la transferencia, para conocer } \\
\text { el sistema y analizar las implicaciones de iniciar el proceso } \\
\text { de transferencia-apropiación, asumiendo los compromisos } \\
\text { derivados a través de acuerdos. }\end{array}$ \\
\hline Segunda & $\begin{array}{l}\text { Comprensión del } \\
\text { funcionamiento del } \\
\text { sistema por parte del grupo } \\
\text { interesado }\end{array}$ & $\begin{array}{l}\text { A partir de los acuerdos de colaboración, los } \\
\text { participantes inician una aproximación al } \\
\text { funcionamiento del Sipia in situ, para comprender las } \\
\text { partes que lo integran, sus interrelaciones y su operación } \\
\text { tanto a nivel técnico como a nivel humano. }\end{array}$ \\
\hline \multirow[t]{2}{*}{ Tercera } & $\begin{array}{l}\text { Planeación de la } \\
\text { implementación del Sipia }\end{array}$ & $\begin{array}{l}\text { Los participantes diseñan y planifican la distribución de } \\
\text { los componentes del sistema, adecuándolo a su contexto } \\
\text { particular. Proceden a su implementación para un primer } \\
\text { ciclo de producción, con un estrecho acompańamiento por } \\
\text { parte de la institución que inició la transferencia. El trabajo } \\
\text { a nivel técnico es tan importante como el desarrollo de las } \\
\text { habilidades para la vida que vaya requiriendo el proceso de } \\
\text { apropiación del Sipia. }\end{array}$ \\
\hline & $\begin{array}{l}\text { Primer ciclo de producción } \\
\text { de manera conjunta }\end{array}$ & $\begin{array}{l}\text { Al finalizar el primer ciclo se reflexiona sobre los logros y } \\
\text { dificultades en esta fase. }\end{array}$ \\
\hline Cuarta & $\begin{array}{l}\text { Segundo ciclo de producción } \\
\text { con acompañamiento }\end{array}$ & $\begin{array}{l}\text { Partiendo de los aprendizajes de la fase anterior, } \\
\text { se inicia el segundo ciclo de producción, con un } \\
\text { acompañamiento menos estrecho, fortaleciéndose así el } \\
\text { proceso de apropiación del sistema. }\end{array}$ \\
\hline Quinta & Consolidación de la T-A & $\begin{array}{l}\text { Los participantes se apropian de la totalidad de los } \\
\text { procesos del Sipia: técnico, pedagógico y de desarrollo } \\
\text { humano, limitando la participación de la institución que } \\
\text { lo transfirió a asesorías puntuales. }\end{array}$ \\
\hline
\end{tabular}

Fuente: elaboración propia

Este modelo se ha implementado en dos sitios: en una institución de formación religiosa del postulantado marista de Haití, durante 2011-2012, y actualmente se encuentra funcionando; y en 
un albergue para adolescentes en riesgo de exclusión social en Yucatán, México, del 2013 al 2014, con resultados de producción aceptables en ese periodo.

\section{En síntesis}

El modelo de transferencia-apropiación de tecnología de un sistema de producción integral de alimentos tiene como meta el desarrollo de habilidades para la vida en los participantes, de forma tal que les permita estar en control de las situaciones cotidianas, favoreciendo su calidad de vida.

Este modelo propicia una transformación en el "ser" de los integrantes de la comunidad de trabajoaprendizaje, es decir, tanto en quienes transfieren como en los que se apropian.

La comunidad de trabajo-aprendizaje se construye a través del análisis reflexivo en torno al proceso de trabajo del Sipia.

La característica esencial de este modelo se basa en la interrelación de los tres ejes que lo conforman: el técnico, el pedagógico y el de desarrollo humano. Los sucesos cotidianos durante el proceso de trabajo-aprendizaje determinan las temáticas de formación para el desarrollo.

El tipo de involucramiento de quienes transfieren se modifica en la medida en que el proceso de apropiación avanza, en función de las necesidades de cada fase del modelo. 


\section{Referencias}

Alfageme, E., Cantos, R. y Martínez, M. (2003). De la participación al protagonismo infantil. Propuestas para la acción. Madrid: Plataforma de Organizaciones de Infancia.

Benbrook, C. (1991). Sustainable Agriculture Research and Education in the Field: A Proceedings. Washington, D.C.: National Academy Press.

Boltvinik, J. (2005). Ampliar la mirada. Un nuevo enfoque de la pobreza y el florecimiento humano. Papeles de Población, 44, 9-42.

Brummett, R. E. y Williams, M. J. (2000). The evolution of aquaculture in African rural and economic development. Ecological Economics, 33, 193-203.

Cassís, A. J. (2011). Donald Schön: una práctica profesional reflexiva en la universidad. Compás Empresarial, 3(5), 14-21.

Cavazos, A. (1997). Un nuevo proyecto de Nación. En La Universidad Marista de Yucatán... una universidad para todos. Mérida Yucatán, México: Centro Marista de Estudios Superiores A.C. Cuadernos Básicos.

Crabay, M. (2010). Adolescencias y juventudes. Subjetividades y riesgos: Contribuciones para su análisis y comprensión. Brujas-Córdoba.

De la Cruz, C. y Sasia, P. (2008). La responsabilidad de la universidad en el proyecto de construcción de una sociedad. Revista Educación Superior y Sociedad, 13(2), 20-51.

Díaz-Barriga, A. (2006). Enseñanza situada: Vinculo entre la escuela y la vida. México, D.F.: McGraw-Hill Interamericana.

Duch, I. (2009). Alternativa pedagógica para la capacitación. San Francisco, CA, Estados Unidos: Creative Commons. Flores, A. (2001). Agro-acuicultura: Principios milenarios aplicados a la producción acuícola sustentable en la actualidad. III Simposio Nacional de Acuicultura y Pesca, Antigua Guatemala (pp. 37-40).

Freire, P. (1973). ¿Extensión o comunicación? La concientización en el medio rural. México, D. F.: Siglo XXI Editores. Gudynas, E. y Acosta, A. (2011). Buen vivir más allá del desarrollo. Qué hacer, 181, 70-81.

Gudynas, E. (2011). Buen vivir: Germinando alternativas al desarrollo. América Latina en Movimiento, ALAI, 462, 1-20.

Isla-Esquivel, M. L., Cuevas-Jiménez, A. y Romero-Yam, L. A. (2011). Factores sociales que afectan el cultivo de tilapia en la Península de Yucatán. Ambiente y Desarrollo, 15(29), 113-136.

Martínez, V. (2014). Habilidades para la vida: una propuesta de formación humana. Itinerario Educativo, 28(63), 61-89.

Max-Neef, M. (1993). Desarrollo a escala humana. Conceptos, aplicaciones y algunas reflexiones. Montevideo: NordanComunidad, Icaria.

Mejía, M. R. y Awad, M. I. (2003). Educación popular hoy. En tiempos de globalización. Bogotá: Ediciones Aurora. Murshed, E., Jahan, K. y Pemsl, D. E. (2011). The impact of integrated aquaculture-agriculture on small-scale farm sustainability and farmers' livelihoods: Experience from Bangladesh. Agricultural Systems, 104(5), 392-402.

Oficina de Derechos Humanos Arzobispado de Guatemala (2004). Construyamos la cultura de paz y el desarrollo local desde nuestra comunidad. Módulo 1. Empoderamiento: Base para el desarrollo local. Guatemala (pp. 2-16).

Organización Mundial de la Salud (oms) (1993). Life Skills Education for Children and Adolescents in Schools. Ginebra: oms, Programme on Mental Health.

Phong, L. T., Udo, H. M. J., Van Mensvoort, M. E. F., Bosma, R. H., QuangTri, L., Nhan D. K. y Van der Zijpp, A. J. (2007). Integrated Agriculture-Aquaculture Systems in the Mekong Delta, Vietnam: An Analysis of Recent Trends. Asian Journal of Agriculture and Development, 4(2), 51-66. 
Porcuna, J. (2007). Producción integrada. Una estrategia de tránsito hacia sistemas más sostenibles. Ecosistemas, 16(1), 37-43

Programa de las Naciones Unidas para el Desarrollo (1990). Human Development Report 1990. Bogotá: Tercer Mundo Editores.

Pullin, R. (2003). Agro-acuicultura integrada y medio ambiente. En Agro-acuicultura integrada: manual básico (pp. 17-18). FAO Documento técnico de pesca nº 407. Roma: FAO.

Roncal Cárdenas, Z. B. (2010). Sistemas integrados de producción sostenible en las microcuencas del flanco OxapampaРиzиzo.

Schön, D. (1992). La formación de profesionales reflexivos: Hacia un nuevo diseño de la enseñanza y el aprendizaje de los profesionales. Barcelona: Paidós.

Sen, A. (2000). Desarrollo y libertad. Buenos Aires: Planeta.

Useche, M. C. y Gelvis Leal, O. J. (2009). Educación, ética y cultura: una mirada desde Paulo Freire. Telos. Revista de Estudios Interdisciplinarios en Ciencias Sociales, 11(2), 182-193. 